\title{
SEMICONTINUITY OF MULTIFUNCTIONS CONNECTED WITH OPTIMIZATION WITH RESPECT TO CONES
}

\author{
ALICJA STERNA-KARWAT \\ (Received 14 February 1984) \\ Communicated by J. H. Rubinstein
}

\begin{abstract}
This paper studies topological upper and lower semicontinuity of the minimal value multifunction and the solution multifunction for optimization problems, which are defined in terms of cones, subject to perturbations in constraints. It extends the results of Tanino and Sawaragi to finite dimensions and one of Berge to multiple objective optimization problems.
\end{abstract}

1980 Mathematics subject classification (Amer. Math. Soc.): 46 A 40, 49 A 50, 49 B 30, 54 C 60, 90 C 30.

\section{Introduction}

Throughout the paper we let $Y$ be a real topological, Hausdorff, vector space.

Definition 1.1. A set $C$ in $Y$ is a cone if $\lambda y \in C$ for all $y \in C$ and $\lambda \geqslant 0$. A convex cone $C$ is one for which $\lambda_{1} y_{1}+\lambda_{2} y_{2} \in C$ for all $y_{1}, y_{2} \in C$ and $\lambda_{1}, \lambda_{2} \geqslant 0$. A pointed cone is one for which $C \cap(-C)=\{0\}$, where 0 denotes the zero element in $Y$.

Definition 1.2. Let $C$ be a pointed cone in $Y$. Then $C$ induces a partial ordering $\leqslant$ in $Y$, namely, for $y_{1}, y_{2} \in Y$ we say $y_{1} \leqslant y_{2}$ if $y_{2}-y_{1} \in C$.

More details about ordered topological vector spaces are in [15].

Definition $1.3[9,14]$. Let $B$ be a subset of $Y$. The point $y_{0} \in B$ is a vector minimal point of $B$ with respect to $C$, denoted $y_{0} \in \min B$, if there exists no $y \in B$ for which $y \leqslant y_{0}$ and $y_{0} \neq y$.

(c) 1986 Australian Mathematical Society $0263-6115 / 86 \$ A 2.00+0.00$ 
Let $B \subset Y ; \bar{B}$ denotes closure in the topology of $Y$, and $B^{c}$ denotes complementation.

Definition 1.4. Let $C$ be a cone in $Y$. A subset $B$ is said to be $C$-semicompact if every open cover of $B$ of the form $\left\{\left(\bar{C}+y_{j}\right)^{c}: y_{j} \in Y, j \in J\right\}$ has a finite subcover.

Let us note that this definition is slightly different to that in [6, Definition 2.5], where the elements $y_{i}$ are assumed to be in $B$. We have decided to change the definition because, according to the previous one, no compact set in the space of real numbers with the usual ordering has such a kind of open cover.

Motivation for the notion of $C$-semicompactness is given in [18].

The following theorem is still valid with our definition of $C$-semicompactness; however, the proof given in [6] has a mistake which can easily be removed, and therefore we do not prove the theorem here.

THEOREM 1.1 [6]. If $C$ is a pointed, convex cone in $Y$, and if $B \subseteq Y$ is a non-empty $(-C)$-semicompact set, then $\min B$ is nonempty.

LEMMA 1.1. Let $C$ and $B$ be as in Theorem 1.1. Then for every $b_{0} \in B$ there is $y \in \min B$ such that $y \leqslant b_{0}$.

Proof. Let us consider a nonempty set $B_{0}=\left\{y \in B: y \leqslant b_{0}\right\}$. If $B_{0} \subset$ $\cup_{j \in J}\left(-\bar{C}+y_{j}\right)^{c}$ for some $y_{j} \in Y$, then $B \subset \cup_{j \in J}\left(-\bar{C}+y_{j}\right)^{c} \cup\left(-\bar{C}+b_{0}\right)^{c} . B$ is $(-C)$-semicompact, so

$$
B \subseteq\left(-\bar{C}+y_{1}\right)^{c} \cup \cdots \cup\left(-\bar{C}+y_{p}\right)^{c} \cup\left(-C+b_{0}\right)^{c}
$$

for some $y_{1}, \ldots, y_{p}$ taken from the set $\left\{y_{j}\right\}_{j \in J}$. Since $B_{0} \cap\left(-\bar{C}+b_{0}\right)^{c}=\varnothing$, we get that

$$
B_{0} \subset \bigcup_{n=1}^{p}\left(-\bar{C}+y_{n}\right)^{c},
$$

which implies that $B_{0}$ is $(-C)$-semicompact. By Theorem 1.1 there exists $y_{0} \in$ $\min B_{0}$, and it can be shown that $y_{0} \in \min B$ as well.

Let $T, X$ be topological Hausdorff spaces, $f: X \rightarrow Y$ a function and $\Gamma: T \rightarrow X$ a multifunction. Consider the family of minimization problems $\left\{P_{t}\right\}_{t \in T}, P_{t}$ : $\min \{f(x): x \in \Gamma(t)\}$, which can be considered as perturbations in constraints from $P_{t_{0}}$ in some neighbourhood of $t_{0}$. Problem $P_{t}$ can be studied from two sides:

(a) we can extend the notion of the minimal value function for $f$ a real-valued function to the minimal value multifunction, namely, $M_{f}: T \rightarrow Y$ will be defined as $M_{f}(t)=\min f(\Gamma(t))$, for $t \in T$; and 
(b) the solution multifunction will be defined as

$$
S_{f}: T \rightarrow X, \quad S_{f}(t)=\left\{x \in \Gamma(t): f(x) \in M_{f}(t)\right\},
$$

for $t \in T$, the set of all solutions to $P_{t}$.

If $f=$ id (the identity function on $X=Y$ ), then $S_{\text {id }}=M_{\text {id }}$, and this multifunction we shall call the minimal multifunction and denote it be $M$; so for a multifunction $\Gamma: T \rightarrow Y$, the minimal multifunction $M: T \rightarrow Y$ is defined by $M(t)=\min \Gamma(t)$ for $t \in T$.

Let us note that $x \in S_{f}(t)$ if and only if there exists no $\bar{x} \in \Gamma(t)$ for which $f(\bar{x}) \leqslant f(x)$ and $f(\bar{x}) \neq f(x)$. This means that $S_{f}(t)$ is the well-known set of Pareto optimal elements for the vector optimization problem $P_{t}$ in Euclidean space.

Optimization with respect to cones and generalizations of Pareto optimality have been studied in a number of papers, $[3,5,7,8,16]$ to name but a few. More references can be found in the above mentioned papers.

Different types of stability of $S_{f}$ and $M_{f}$, when $Y=R$ and $C=R_{+}$is the nonnegative half line, have been studied for years. An extensive bibliography on this subject is given in [1].

Let us recall the definitions of topological lower and upper semicontinuity.

Definition 1.5. A multifunction $\Gamma: T \rightarrow X$ is said to be lower semicontinuous (l.s.c.) at a point $t_{0} \in T$ if for every open set $Q$ which meets $\Gamma\left(t_{0}\right)$ there exists a neighbourhood $V$ of $t_{0}$ such that $Q$ meets $\Gamma(t)$ for every $t \in V$.

A multifunction $\Gamma: T \rightarrow X$ is said to be upper semicontinuous(u.s.c.) at $t_{0}$ if for every open set $Q$ which includes $\Gamma\left(t_{0}\right)$ there exists a neighbourhood $V$ of $t_{0}$ such that $Q$ includes $\Gamma(t)$ for every $t \in V$.

We say that $\Gamma$ is continuous at $t_{0}$ if it is both 1.s.c. and u.s.c. at $t_{0}, \Gamma$ is continuous if it is continuous at every point $t \in T$.

\section{Stability conditions for the minimal value multifunction and the solution multifunction}

Denote by $R^{n}$ the $n$-dimensional Euclidean space ordered by the nonnegative orthant $R_{+}^{n}$.

The well known Berge's theorem states: if $X, T$ are topological spaces, and if $\Gamma$ : $T \rightarrow X$ is a continuous multifunction such that for every $t \in T, \Gamma(t)$ is nonempty 
and compact, then for every continuous, real-valued function $f: X \rightarrow R^{1}$

(i) the minimal value function $M_{f}$ is continuous,

(ii) the solution multifunction $S_{f}$ is u.s.c.

The following simple example in $R^{2}$ shows that the solution multifunction $S_{\text {id }}$ may not be u.s.c., when $\Gamma$ is continuous and compact valued unless the pair $(Y, C)=\left(R, R_{+}\right)$, and therefore Berge's theorem ceases to be valid in the general case.

EXAmple 2.1. Let $\Gamma:[0,1] \rightarrow R^{2}$ be defined as follows: $\Gamma(t)=$ the closed line segment between $(-1+t, t)$ and $(0,0)$ for $0 \leqslant t \leqslant 1$, so that $\Gamma$ is compact valued and continuous. Then

$$
S_{\mathrm{id}}(t)=\min \Gamma(t)= \begin{cases}(-1,0), & t=0, \\ \Gamma(t), & 0<t<1, \\ (0,0), & t=1\end{cases}
$$

Hence $S_{\text {id }}$ is not u.s.c. at 0 and at 1 , and thus $M_{\text {id }}$ is not continuous as well.

But we can state the theorem for lower semicontinuity of the set of vector minimal elements for $\Gamma$ as follows:

THEOREM 2.1. Let $T$ be a topological Hausdorff space, and let $Y$ be a real topological Hausdorff vector space, ordered by a pointed, convex closed cone $C$. Let $t_{0}$ be a point in $T$ and $\Gamma: T \rightarrow Y$ a multifunction continuous at $t_{0}$. Then the minimal multifunction

$$
M: T \rightarrow Y, \quad M(t)=\min \Gamma(t) \quad \text { for } t \in T
$$

is l.s.c. at $t_{0}$,

(a) if $\Gamma(t)$ is nonempty, $(-C)$-semicompact and closed in some neighbourhood of $t_{0}$, and $\Gamma\left(t_{0}\right)$ is compact, or (b) if $T$ fulfills the first countability axiom at $t_{0}, \Gamma(t)$ is nonempty and $(-C)$-semicompact in some neighbourhood of $t_{0}$, and $\Gamma\left(t_{0}\right)$ is compact.

Proof. Let $A$ be a linear ordered set, and let $\left\{V_{\alpha}\right\}_{\alpha \in A}$ be a nested base for the neighbourhood system of the point $t_{0}$. We can assume that $\Gamma$ fulfills all assumptions of the theorem in every $V_{\alpha}$.

Suppose $M$ is not 1.s.c. at $t_{0}$. Then we can find $y_{0} \in M\left(t_{0}\right)$ and its neighbourhood $Q$ in $Y$, and a net $\left\{t_{\alpha}\right\}_{\alpha \in A}$ converging to $t_{0}$, such that

$$
t_{\alpha} \in V_{\alpha} \text { for every } \alpha \in A
$$


and

$$
M\left(t_{\alpha}\right) \cap Q=\varnothing .
$$

Let $\mathscr{U}(0)$ be a local base in $Y$. Let us denote by $\left\{U^{\prime}\right\}_{U \in \mathscr{U}(0)}$ a base of the neighbourhood system of the point $y_{0}$ of the kind

$$
U^{\prime}=\left(y_{0}+U\right) \cap Q \text { for } U \in \mathscr{U}(0) .
$$

Fix $U^{\prime}$; since $y_{0} \in \Gamma\left(t_{0}\right) \cap U^{\prime}$ and $\Gamma$ is 1.s.c. at $t_{0}$, we can find elements $\alpha\left(U^{\prime}\right) \in A$ and $y_{\alpha\left(U^{\prime}\right)} \in \Gamma\left(t_{\alpha\left(U^{\prime}\right)}\right) \cap U^{\prime}$. Let us note that the net $\left\{y_{\alpha\left(U^{\prime}\right)}\right\}_{U \in \mathscr{L}(0)}$ converges to $y_{0}$.

Define a set

$$
\mathscr{U}(A)=\left\{\alpha\left(U^{\prime}\right): U \in \mathscr{U}(0)\right\} \subseteq A .
$$

The first case is when $\mathscr{U}(A)$ is a cofinal set in $A$. Let us note that for part (b) of Theorem 2.1 we can choose $\mathscr{U}(A)$ cofinal; and the closedness of $\Gamma(t)$ will not be necessary when $\mathscr{U}(A)$ is a cofinal set, so the first case establishes the part (b) of the theorem.

In this case $\left\{t_{\alpha\left(U^{\prime}\right)}\right\}_{U \in \mathscr{U}(0)}$ is a subnet of $\left\{t_{\alpha}\right\}_{\alpha \in A}$, so it converges to $t_{0}$ as well. By Lemma 1.1, one actually can find $z_{\alpha\left(U^{\prime}\right)} \in M\left(t_{\alpha\left(U^{\prime}\right)}\right)$ such that $z_{\alpha\left(U^{\prime}\right)} \leqslant y_{\alpha\left(U^{\prime}\right)}$ for every $\alpha\left(U^{\prime}\right) \in \mathscr{U}(A)$.

Next, for every $U \in \mathscr{U}(0)$ let us define open sets

$$
G_{U}=\Gamma\left(t_{0}\right)+U
$$

By upper semicontinuity of $\Gamma$ at $t_{0}$, we obtain $\beta(U) \in A$ such that $\Gamma(t) \subseteq G_{U}$ for every $t \in V_{\beta(U)}$.

Now we will construct a new set $\{\gamma(U)\}_{U \in \mathscr{U}(0)}$. Take $U \in \mathscr{U}(0)$;

(i) if $\beta(U) \leqslant \alpha\left(U^{\prime}\right)$ we put $\gamma(U)=\alpha\left(U^{\prime}\right)$,

(ii) if $\beta(U)>\alpha\left(U^{\prime}\right)$ we can find $\alpha \in \mathscr{U}(A)$ such that $\alpha \geqslant \beta(U)$ because $\mathscr{U}(A)$ is a cofinal set in $A$, and then put $\gamma(U)=\alpha$. The set $\{\gamma(U)\}_{U \in \mathscr{U}(A)}$ is a cofinal in $\mathscr{U}(A)$. Indeed, if there exists $\alpha\left(U_{0}^{\prime}\right) \in \mathscr{U}(A)$, such that $\alpha\left(U_{0}^{\prime}\right)>\gamma(U)$ for every $U \in \mathscr{U}(0)$, then we consider $\gamma\left(U_{0}\right)$; we have $\alpha\left(U_{0}^{\prime}\right)>\gamma\left(U_{0}\right)$, and by the definition of the set $\{\gamma(U)\}_{U \in \mathscr{Q}(0)}$ it must actually be true that $\beta\left(U_{0}\right)>\alpha\left(U_{0}^{\prime}\right)$, whence

$$
\gamma\left(U_{0}\right) \geqslant \beta\left(U_{0}\right)>\alpha\left(U_{0}^{\prime}\right)>\gamma\left(U_{0}\right),
$$

which is a contradiction. Since $\gamma(U) \geqslant \beta(U)$, so $V_{\gamma(U)} \subseteq V_{\beta(U)}$, and therefore $\Gamma(t) \subseteq G_{U}$ for every $t \in V_{\gamma(U)}$. Let us observe that $z_{\gamma(U)} \in M\left(t_{\gamma(U)}\right)$ for every $U \in \mathscr{U}(0)$, because $\gamma(U) \in \mathscr{U}(A)$. Hence

$$
z_{\gamma(U)} \in G_{U}=\Gamma\left(t_{0}\right)+U \text { for every } U \in \mathscr{U}(0)
$$

and $z_{\gamma(U)}$ can be written

$$
z_{\gamma}(U)=x_{U}+u \text { for some } x_{U} \in \Gamma\left(t_{0}\right) \text { and } u \in U \text {. }
$$

Since $\Gamma\left(t_{0}\right)$ is a compact set, we can choose a subnet of $\left\{x_{U}\right\}_{U \in \mathscr{U}(0)}$, which is convergent to $x_{0} \in \Gamma\left(t_{0}\right)$, and consequently there exists a subnet of $\left\{z_{\gamma(U)}\right\}_{U \in \mathscr{U}(0)}$ which is convergent to $x_{0}$. By the definition, $z_{\gamma(U)} \leqslant y_{\gamma(U)}$, and $C$ is closed, so 
$x_{0} \leqslant y_{0}$, whence $x_{0}=y_{0}$. Therefore

$$
M\left(t_{\gamma(U)}\right) \cap Q \neq \varnothing \quad \text { for some } U \in \mathscr{U}(0),
$$

which is a contradiction.

The second case is when $\mathscr{U}(A)$ is not a cofinal set in $A$. The theorem is true if $t_{0}$ is an isolated point; suppose $t_{0}$ is not an isolated point. $T$ is a Hausdorff space, so for every $\alpha \in A$ there exists $\gamma \in A$ such that $\gamma>\alpha$. Indeed, if there is a $\alpha \in A$ such that $\gamma \geqslant \alpha$ for any $\alpha \in A$, then we have $V_{\gamma} \subseteq V_{\alpha}$ for $\alpha \in A$. The point $t_{0}$ is not isolated, so we get $t_{1} \in V_{\gamma}$ such that $t_{1} \neq t_{0}$. T is a Hausdorff space, so there exist open sets $U_{0}$ and $U_{1}$ such that $t_{0} \in U_{0}, t_{1} \in U_{1}$ and $U_{0} \cap U_{1}=\varnothing .\left\{V_{\alpha}\right\}_{\alpha \in A}$ is a neighbourhood system of $t_{0}$, so we can find $\alpha \in A$ for which $V_{\alpha} \subseteq U_{0}$. Therefore $t_{1} \in V_{\gamma} \subseteq U_{0}$, which is a contradiction.

Since $\mathscr{U}(A)$ is not a cofinal set now, there exists $\alpha_{0} \in A$ such that $\alpha_{0}>\alpha\left(U^{\prime}\right)$ for every $U \in \mathscr{U}(0)$. Then

$$
V_{\alpha} \subseteq V_{\alpha_{0}} \subseteq V_{\alpha\left(U^{\prime}\right)} \text { for every } U \in \mathscr{U}(0) \text { and every } \alpha \geqslant \alpha_{0}
$$

and

$\Gamma(t) \cap U^{\prime} \neq \varnothing$ for every $t \in V_{\alpha}, \quad$ for every $\alpha \geqslant \alpha_{0}$ and for every $U \in \mathscr{U}(0)$.

Hence, for every $\alpha \geqslant \alpha_{0}$ and for every $U \in \mathscr{U}(0)$ we can take $y_{\alpha}^{U} \in \Gamma\left(t_{\alpha}\right) \cap U^{\prime}$. Fix $\alpha \geqslant \alpha_{0}$; the net $\left\{y_{\alpha}^{U}\right\}_{U \in \mathscr{U}(0)}$ is convergent to $y_{0}$, and $\Gamma\left(t_{\alpha}\right)$ is closed, so $y_{0} \in \Gamma(t)$. By Lemma 1.1 we get $z_{\alpha} \in M\left(t_{\alpha}\right)$ such that $z_{\alpha} \leqslant y_{0}$. Consider the net $\left\{t_{\alpha}\right\}_{\alpha \geqslant \alpha_{0}}$. Exactly as in the first case, we find a subnet of $\left\{z_{\alpha}\right\}_{\alpha \geqslant \alpha_{0}}$ which is convergent to $y_{0}$.

This contradiction establishes lower semicontinuity of $M$.

Corollary 2.1. Let $T$ and $Y$ be as in Theorem 2.1. Let $\Gamma: T \rightarrow Y$ be $a$ continuous multifunction with $\Gamma(t)$ nonempty and compact for every $t \in T$. Then the minimal $M$ is l.s.c. at every point $t \in T$.

TheOREM 2.2. Let $T$ and $Y$ be as in Theorem 2.1. Let a multifunction $\Gamma: T \rightarrow X$ be continuous at $t_{0}$ with $\Gamma(t)$ nonempty and compact in some neighbourhood of $t_{0}$. Then for every function $f: X \rightarrow Y$ which is continuous in some open set including $\Gamma\left(t_{0}\right)$, the minimal value multifunction $M_{f}$ is l.s.c. at $t_{0}$.

Proof. Let $f, \Gamma$ be as in the hypothesis: Then $f \Gamma: T \rightarrow Y$, defined by $(f \Gamma)(t)=f(\Gamma(t))$ for every $t \in T$, fulfills all of the assumptions of Theorem 2.1. Hence $M_{f}$, which is the minimal multifunction for $f \Gamma$, must be l.s.c. at $t_{0}$.

Theorem 2.2 extends part (i) of Berge's theorem to vector optimization, because for a real valued function $f$ the multifunction $M_{f}$ is actually a function and its lower semicontinuity as a multifunction is equivalent to the continuity. 
THEOREM 2.3. Let a multifunction $\Gamma: T \rightarrow X$ be continuous at $t_{0}$ and $f: X \rightarrow Y$ be a continuous and one-to-one function in some open set $G$ including $\Gamma\left(t_{0}\right)$. Then the solution multifunction $S_{f}: T \rightarrow X$ is l.s.c. at $t_{0}$

(a) if $\Gamma(t)$ is nonempty and compact in some neighbourhood of $t_{0}$, and the function $f$ is open [13] in $G$, or

(b) if $T$ is first countable at $t_{0}, X$ is a vector topological space with a countable local base, $f(\Gamma(t))$ is $(-C)$-semi-compact in some neighbourhood of $t_{0}$, and $\Gamma\left(t_{0}\right)$ is a compact set.

Proof. (a) Let $Q$ be an open set in $X$, and let $x_{0} \in S_{f}\left(t_{0}\right) \cap Q$. Since $f$ is open in $G$, then $f(Q \cap G)$ is an open set and $f\left(x_{0}\right) \in M_{f}\left(t_{0}\right) \cap f(Q \cap G)$. By Theorem 2.2, $M_{f}$ is 1.s.c. at $t_{0}$, thus there is a neighbourhood $V$ of $t_{0}$ such that $M_{f}(t) \cap$ $f(Q \cap G) \neq \varnothing$ for every $t \in V$. Let $y_{t} \in M_{f}(t) \cap f(Q \cap G)$ for $t \in V$. Hence there exist elements $x_{t} \in \Gamma(t)$ and $q_{t} \in Q \cap G$ such that

$$
f\left(x_{t}\right)=y_{t}=f\left(q_{t}\right) \text { for } t \in V,
$$

and one must actually have $x_{t}=q_{t}$ for some neighbourhood $V_{1}$ of $t_{0}$ contained in $V$ because $f$ is one-to-one in $G$ and $\Gamma$ is u.s.c. at $t_{0}$. Thus $x_{t} \in \Gamma(t) \cap f^{-1}\left(M_{f}(t)\right)$ $\cap Q \cap G$, and therefore $S_{f}(t) \cap Q \neq \varnothing$ for every $t \in V_{1}$, which proves the lower semicontinuity of $S_{f}$ at $t_{0}$.

(b) Suppose that $S_{f}$ is not l.s.c. at $t_{0}$. Because of the assumptions imposed on the spaces $T$ and $X$, this means that there exist a sequence $\left\{t_{n}\right\}_{n=1}^{\infty}$ converging to $t_{0}$ and a point $x_{0} \in S_{f}\left(t_{0}\right)$ and its neighbourhood $Q$ such that $S_{f}\left(t_{n}\right) \cap Q=\varnothing$ for $n=1,2, \ldots$ Since $\Gamma$ is 1.s.c. at $t_{0}$, we can find $\{x\}_{n=1}^{\infty}$ tending to $x_{0}$ such that $x_{n=1} \in \Gamma\left(t_{n}\right)$ for $n>N$. By Lemma 1.1, we get elements $a_{n} \in \Gamma\left(t_{n}\right)$ with $f\left(a_{n}\right) \in$ $M_{f}\left(t_{n}\right)$ and $f\left(a_{n}\right) \leqslant f\left(x_{n}\right)$ for $n>N$.

Let $\left\{U_{k}\right\}_{k=1}^{\infty}, U_{k+1} \subset U_{k}$, a local base in $X$. Consider the open sets

$$
G_{k}=\Gamma\left(t_{0}\right)+U_{k} \text { for } k=1,2, \ldots
$$

By upper semicontinuity at $t_{0}$ of $\Gamma$ we must actually have $\Gamma\left(t_{n_{k}}\right) \subset G_{k}$ for some subsequence $\left\{t_{n_{k}}\right\}_{k=1}^{\infty}$ of $\left\{t_{n}\right\}_{n=1}^{\infty}$. Thus $a_{n_{k}} \in C_{k}$ for $k=1,2, \ldots$, and since $\Gamma\left(t_{0}\right)$ is compact, a sequence $\left\{a_{n_{k}}\right\}_{k=1}^{\infty}$ possesses a convergent subsequence. Let $\left\{a_{n_{k}}\right\}_{k=1}^{\infty}$ itself tend to $a_{0} \in \Gamma\left(t_{0}\right)$. Let us observe that $a_{n_{k}}, x_{n_{k}} \in G$ for $k>K$ because $\Gamma$ is u.s.c. at $t_{0}$. The function $f$ is continuous in $G, f\left(a_{n}\right) \leqslant f\left(x_{n}\right)$ for $n>N$, and so $f\left(a_{0}\right) \leqslant f\left(x_{0}\right)$. Since $f\left(x_{0}\right) \in M_{f}\left(t_{0}\right)$, one must actually have $f\left(a_{0}\right)=f\left(x_{0}\right)$. Both $a_{0}$ and $x_{0}$ belong to $G, f$ is one-to-one in $G$, and so $a_{0}=x_{0}$. Hence $a_{n_{k}} \in S_{f}\left(t_{n_{k}}\right) \cap Q$ for some $k$, which is a contradiction.

Let us note that the assumption given for $X$ in part (b) of Theorem 2.3 is equivalent to the assumption that $X$ is metrizable [12, page 49].

Theorems 2.1 and 2.3 are generalizations of results of Tanino and Sawaragi [17, Theorems 5.2 and 7.2, respectively] for infinite dimensions as well as an extension to a larger class of multifunctions. Tanino and Sawaragi have used slightly 
different sequential definitions of semicontinuities of multifunctions which, however, imply our definitions under their assumptions about the spaces ( $T$ is such that the concept of convergence is defined in terms of sequences, and $Y$ is a Euclidean space), and with the additional condition imposed on $\Gamma$ that $U_{t \in V} \Gamma(t)$ be compact for some neighbourhood $V$ of $t_{0}$. For details of the different kinds of semicontinuity of multifunctions see $[2,4,10,11,13]$. The following example shows a multifunction $\Gamma$ with values in a Banach space which fulfills all of the assumptions of Theorem 2.1, but fails to satisfy one of Theorem 5.2 [17].

EXAmple 2.2. Let $Y$ be the space of continuous real functions on the interval $[0,1]$ with the sup norm, ordered by a closed, convex, pointed cone (even with nonempty interior)

$$
C=\{f: f(t) \geqslant 0 \text { for every } t \in[0,1]\} .
$$

Define a multifunction $\Gamma: \cup_{n=1}^{\infty}\{1 / n\} \cup\{0\} \rightarrow Y$ by

$$
\Gamma(0)=\{0\}, \quad \Gamma\left(\frac{1}{n}\right)=B\left(0, \frac{1}{n}\right) \cap C \text { for } n=1,2, \ldots,
$$

where $B(0,1 / n)$ denotes a closed ball centred at 0 with radius $1 / n$. It is easy to see that $\Gamma$ satisfies every assumption of Theorem 2.1 except that, for any natural number $m$, the set

$$
\overline{\bigcup_{n \geqslant m} \Gamma\left(\frac{1}{n}\right)}=B\left(0, \frac{1}{m}\right) \cap C
$$

is not a compact set, and thus we cannot apply either Theorem 5.2 or Theorem 7.2 of [17] to $\Gamma$.

Another remark on the paper of Tanino and Sawaragi [17] is that they consider variations in the domination structure $D$, which is a generalization of a notion of the order in a partially ordered space. Keeping the notation as in [17], we can extend the definition of $(-C)$-semicompactness to the case of the domination structure $D$ by declaring that $B \subset Y$ is $D$-semicompact if every open cover of $B$ of the form $\left\{\left(y_{j}+\overline{D\left[y_{j}\right]}\right)^{c}: y_{j} \in Y, j \in J\right\}$ has a finite subcover.

Under the assumption about $D$ in [17], and after replacing ( $-C)$-semicompactness by $D$-semicompactness, Theorem 1.1 as well as our results in this paper remain true, and the proofs are in fact the same. However, this approach is not under consideration here, and for details of this subject the reader is referred to [17].

\section{Examples}

The following examples show that the assumptions of Theorem 2.1 cannot be weakened. 
EXAmple 3.1. We define a continuous multifunction $\Gamma$ with $\Gamma(t)$ closed and bounded for every $t$ and $\Gamma\left(t_{0}\right)$ compact for some $t_{0}$, but such that $M$ is not 1.s.c. at $t_{0}$. Namely, let us take as $Y$ the Banach space of all sequences of real numbers converging to 0 , with the sup norm, and let us order it by the pointed, closed, convex cone $C$ of all nonnegative sequences. Define $\Gamma:[0,1] \rightarrow Y$ by .

$$
\Gamma(t)=\{y:\|y\| \leqslant t\} .
$$

Then

$$
M(t)=\min \Gamma(t)=\varnothing \quad \text { for } t \neq 0 \text { (for details see [6]), }
$$

and

$$
M(0)=\min \Gamma(0)=\{0\}
$$

whence $M$ is not l.s.c. at 0 .

EXAMPLE 3.2. We cannot reject the assumption that $\Gamma$ is u.s.c. at $t_{0}$ in Theorem 2.1. Indeed, let us define an 1.s.c. multifunction

$$
\begin{aligned}
& \Gamma:\{t: t \geqslant 0\} \rightarrow R^{2} \text { by } \\
& \Gamma(t)= \begin{cases}I \cup\{(-1 / t, 0)\}, & t>0, \\
I, & t=0,\end{cases}
\end{aligned}
$$

where $I=\{(-x, x): 0 \leqslant x \leqslant 1\}$. Then $\Gamma$ is compact valued but is not u.s.c. at 0 , and so

$$
M(t)= \begin{cases}I, & t=0, \\ \{(-1 / t, 0)\}, & 0<t \leqslant 1, \\ \{(-x, x): 1 \leqslant x<1 / t\} \cup\{(-1 / t, 0)\}, & t>1,\end{cases}
$$

is not 1.s.c. at 0 .

This example also shows that the minimal value function $M_{f}$ may not be 1.s.c. when $f$ is continuous, and when $\Gamma$ is l.s.c. and compact valued.

The next example proves that we cannot omit the assumption about $(-C)$-semicompactness for $\Gamma(t)$ in some neighbourhood of $t_{0}$ in Theorem 2.1(b).

EXAMPLE 3.3. Let $\Gamma:[0,1] \rightarrow R^{2}$ be the continuous multifunction defined by

$$
\Gamma(t)= \begin{cases}\{(x, y: 1-x<y \leqslant 2-x\} \cup\{(1,0)\}, & 0<t \leqslant 1, \\ \{(x, y: 1-x \leqslant y \leqslant 2-x\}, & t=0 .\end{cases}
$$

Then

$$
M(t)= \begin{cases}\{(1,0)\}, & 0<t \leqslant 1, \\ \{(x, 1-x): 0 \leqslant x \leqslant 1\}, & t=0,\end{cases}
$$

is not l.s.c. at 0 , although $\Gamma(0)$ is compact. 
Now we show that in Theorem 2.1(b), $M$ may not be l.s.c. at $t_{0}$ when $\Gamma\left(t_{0}\right)$ is not a compact set.

EXAMPLE 3.4. Let $\Gamma:[0,1] \rightarrow R^{2}$ be the continuous multifunction defined by

$$
\begin{aligned}
& \Gamma(t)=\{(1, t)\} \cup\left\{\left(-\frac{1}{x}, x\right): t \leqslant x \leqslant 1\right\}, \\
& \Gamma(0)=\{(1,0)\} \cup\left\{\left(-\frac{1}{x}, x\right): 0<x \leqslant 1\right\} .
\end{aligned}
$$

Then $\Gamma(t)$ is $\left(-R_{+}^{2}\right)$-semicompact for every $t \neq 0, \Gamma(0)$ is closed, and $\min \Gamma(0) \neq$ $\varnothing$, but

$$
M(t)= \begin{cases}\{(1,0)\}, & t=0 \\ \left\{\left(-\frac{1}{t}, t\right)\right\}, & t \neq 0\end{cases}
$$

is not 1.s.c. at 0 .

As a final example, it will be recalled that we cannot obtain lower semicontinuity of $S_{f}$ when $f$ is a continuous function, even when $f$ is linear and real valued, and $\Gamma$ is convex and compact valued. Indeed, let us define a continuous multifunction $\Gamma:[0,1] \rightarrow R^{2}$ by

$$
\Gamma(t)= \begin{cases}I \cap B(t), & t \neq 0 \\ I, & t=0\end{cases}
$$

where $B(t)$ denotes the closed ball centred at $(0,1 / t)$ and with radius $1 / t$ for $t \neq 0$, and where $I=\{(x, y): x, y \in[0,1]\}$.

Let $f: R^{2} \rightarrow R^{1}$ be defined by $f(x, y)=y$ for $(x, y) \in R^{2}$. Then $M_{f}(t)=0$ for every $t$, and

$$
\begin{aligned}
S_{f}(t) & =\{(x, y) \in \Gamma(t): y=0\} \\
& = \begin{cases}\{(0,0)\}, & t \neq 0, \\
\{(x, 0): 0 \leqslant x \leqslant 1\}, & t=0,\end{cases}
\end{aligned}
$$

is not 1.s.c. at $t=0$.

\section{References}

[1] E. Bednarczuk, 'On upper semicontinuity of global minima in constrained optimization problems', J. Math. Anal. Appl. 86 (1982), 309-318.

[2] C. Berge, Topological spaces (Macmillan Company, New York, 1963).

[3] J. Borwein, Optimization with respect to partial orderings (PhD Thesis, Oxford University, 1974).

[4] J. Borwein, 'Convex relations in analysis and optimization', pp. 335-377 in Generalized concavity in optimization and economics, eds. S. Schaible and W. T. Ziemba (Academic Press, London, 1981). 
[5] L. Cesari and M. B. Suryanarayna, 'Existence theorems for Pareto optimization; Multivalued and Banach space valued functionals', Trans. Amer. Math. Soc. 224 (1978), 37-65.

[6] H. W. Corley, 'An existence result for minimizations with respect to cones', J. Optim. Theory Appl. 31 (1980), 277-281.

[7] H. W. Corley, 'Duality theory for maximization with respect to cones', J. Math. Anal. Appl. 84 (1981), 560-568.

[8] B. D. Craven, 'Strong vector minimization and duality', Z. Angew. Math. Phys. 60 (1980), 1-5.

[9] B. D. Craven, 'Vector-valued optimization in generalized concavity', pp. 661-687 in Optimization and economics, eds. S. Schaible and W. T. Ziemba (Academic Press, London, 1981).

[10] J. P. Delahaye and J. Denel, 'The continuities of the point-to-set maps, definitions and equivalences', Math. Programming Stud. 10 (1979), 8-12.

[11] W. Hogan, 'Point-to-set maps in mathematical programming', SIAM Rev. 15 (1973), 591-603.

[12] J. L. Kelly, Linear topological spaces (Van Nostrand, Princeton, N.J., 1963).

[13] K. Kuratowski, Topology (Academic Press, New York and Polish Scientific Publishers, Warszawa, 1966).

[14] J. G. Lin, 'Maximal vectors and multi-objective optimization', J. Optim. Theory Appl. 18 (1976), $41-64$.

[15] A. L. Peressini, Ordered topological vector spaces (Harper and Row, New York, Evanston, London, 1967).

[16] S. Rolewicz, 'On sufficient conditions of vector optimization', Methods of Oper. Res. 43 (1981), $151-156$.

[17] T. Tanino and Y. Sawaragi, 'Stability of non dominated solutions in multicriteria decision-making', J. Optim. Theory. Appl. 30 (1980), 229-253.

[18] D. H. Wagner, 'Semi-compactness with respect to Euclidean cone', Canad. J. Math. 29 (1977), 29-36.

\section{Department of Mathematics}

Monash University

Clayton, Victoria 3168

Australia 